\title{
CONFINEMENT EFFECTS ON THE LOCAL MOTION IN NANOCOMPOSITES
}

\author{
S. H. ANASTASIADIS ${ }^{*}$, , K. KARATASOS ${ }^{*}, \dot{y}$, G. VLACHOS* ${ }^{*}$ E. MANIAS**,\#, and \\ E. P. GIANNELIS**
}

* Foundation for Research and Technology-Hellas, Institute of Electronic Structure and Laser, P. O. Box 1527, 71110 Heraklion, Crete, Greece

** Cornell University, Department of Materials Science and Engineering, Ithaca, NY 14853, U.S.A.

\begin{abstract}
The local segmental dynamics of polymers confined within the 15-20 ${ }^{1}$ interlayer spacing of nanocomposites consisting of poly(methyl phenyl siloxane) intercalated within organically modified silicates, has been investigated utilizing Dielectric Relaxation Spectroscopy. The effect of confinement on the local reorientational dynamics is evident by the observation of a relaxation mode, which is much faster than the segmental $\alpha$-relaxation of the bulk polymer and exhibits much weaker temperature dependence. This is attributed to the restrictions placed by the interlayer spacing on the cooperative volume required for the $\alpha$-relaxation.
\end{abstract}

\section{INTRODUCTION}

One promising way to synthesize polymer nanocomposites is by intercalating polymers in layered inorganic hosts. Layered-silicate based polymer nanocomposites have become an attractive set of organic-inorganic hybrid materials because of their obvious potential as technological materials [1-6].

Beyond the conventional phase separated polymer/silicate nanocomposites, for which the polymer and the inorganic host remain immiscible, two types of hybrids are possible: (i) intercalated, in which a single extended polymer chain is intercalated between the host layers resulting in a well ordered multilayer with alternating polymer/inorganic layers and a repeat distance of a few nm; (ii) exfoliated or delaminated, in which the silicate layers (1nm thick) are exfoliated and dispersed in a continuous polymer matrix. The intercalated polymer/silicate nanocomposites offer a unique avenue for studying the static and dynamic behavior of small molecules and macromolecules in nanoscopic confinements. Actually, in these model systems, one can utilize conventional analytical techniques (like thermal analysis, NMR, dielectric spectroscopy, inelastic neutron scattering, rheology) on macroscopic samples and, nevertheless, study the properties of 2-3 nm thick polymer films [7].

The dynamics in thin polymer films and of small molecules and macromolecules in porous media has recently attracted the interest of the scientific community. For thin polymer films, reports of both enhanced and reduced mobility relative to the bulk have been reported. It appears that the effect of a free surface is to decrease the glass transition temperature $\left(T_{g}\right)$ and, thus, to enhance the mobility [8,9], due a decrease in density [10]. However, the effect of an attractive wall is to increase the $T_{g}$ thus reducing the mobility $[11,12,13]$. Computer simulations showed that the effect of confinement even with neutral walls is to reduce the mobility due to an

$\dagger \quad$ Also at University of Crete, Physics Department, 71003 Heraklion Crete, Greece

ý Present Address: University of Leeds, Department of Physics and Astronomy, Leeds LS2 9JT, U. K.

\# Present Address: Pennsylvania State University, Department of Materials Science and Engineering, State College, PA 16802, U.S.A. 
increase in density [14]. For free standing thin films, $T_{g}$ was found to decrease linearly with thickness [15]. Moreover, finite size effects were favorable for the depression of the 1st-order phase transitions, leading to a considerable shift of the freezing point to far below the appropriate solidification temperature of bulk liquids [16]. In the case of confinement in porous media, the spin-lattice NMR relaxation times of small organic molecules are significantly shorter than for the bulk liquids with the effect depending on the organic/silica interactions $[17,18]$. The issue of a characteristic length scale of cooperativity, which determines the dynamic glass transition, has been raised following studies by solvation dynamics [19] and dielectric spectroscopy [20-23], with the data showing slightly slower [19,21,23] or slightly faster [20,24] dynamics for the fluid in the pores than in the bulk. The effects of coating the pore surface on the dynamics was also investigated $[21,22]$.

In this manuscript, a dielectric relaxation spectroscopy investigation is presented attempting to probe the role of confinement on local dynamics utilizing a series of intercalated polymer/silicate nanocomposites. The organically-modified layered silicates were mixed with low-molecular weight poly(methyl phenyl siloxane), PMPS, whose segmental motion is dielectrically active. PMPS is confined within the 15-20 1 interlayer spacing of the nanocomposites. A relaxation mode, which is much faster than the segmental $\alpha$-relaxation of the bulk polymer, is consistently observed with much weaker temperature dependence. This should be attributed to the restrictions placed by the interlayer spacing on the cooperative volume required for the $\alpha$-relaxation.

\section{EXPERIMENTAL SECTION}

\section{Materials}

Organically modified layered silicates (a hectorite and a bentonite) were synthesized by a cation exchange reaction between the layered silicate hosts and excess dioctadecyl-dimethylammonium bromide, as outlined previously [25], in order to render the originally hydrophilic silicate surface organophilic. Hybrids were prepared by mixing dry organosilicate and poly(methyl phenyl siloxane), PMPS, $\left(M_{w}=2600, M_{w} / M_{n}=1.20\right)$. Various percentages of PMPS were used (Table I) in order to span the range from starved to just over full galleries. The materials were allowed sufficient time $\sim 60$ úC under ultrasonication for the PMPS chains to diffuse into the organically modified silicates (PMPS is liquid at room temperature). The hybrids as well as the silicates themselves were characterized by $\mathrm{x}$-ray diffraction (Scintag Inc. $\theta-\theta$ diffractometer equipped with a Germanium detector using $\mathrm{Cu} \mathrm{K} \alpha$ radiation) in order to determine the interlayer spacing before and after intercalation. The data are shown in Table I.

\begin{tabular}{|c|c|c|c|c|}
\hline Code & Silicate & Polymer & wt\% Polymer & $\mathrm{d}_{100}$ spacing \\
\hline B34 & B34 & - & - & $23^{1}$ \\
\hline B38 & B38 & - & - & $25^{1}$ \\
\hline $15 \%$ & B34 & PMPS & 15 & $33^{1}\left(^{*}\right)$ \\
\hline $20 \%$ & B34 & PMPS & 20 & $32^{1}$ \\
\hline $25 \%$ & B38 & PMPS & 25 & $36^{1}$ \\
\hline $30 \%$ & B34 & PMPS & 30 & $34^{1}$ \\
\hline $\begin{array}{l}\text { * A shoulder is present at } 23^{1} \\
\text { i.e., a starved system }\end{array}$
\end{tabular}




\section{Dielectric Spectroscopy}

Dielectric relaxation spectroscopy, DS, was used to investigate the collective segmental dynamics of PMPS as a function of temperature. The complex dielectric permitivity $\varepsilon^{*}(\omega)=\varepsilon^{\prime}(\omega)-i \varepsilon^{\prime \prime}(\omega)$ of a macroscopic system is given by the one-sided Fourier transform of the time derivative of the normalized response function $\mathrm{C}(\mathrm{t})$

$$
\varepsilon^{*}(\omega)-\varepsilon_{\infty}=-\Delta \varepsilon \int_{0}^{\infty}[d C(t) / d t] \exp (-i \omega t) d t
$$

where $i^{2}=-1, \Delta \varepsilon=\varepsilon_{0}-\varepsilon_{\infty}$ is the relaxation strength with $\varepsilon_{0}$ and $\varepsilon_{\infty}$ being the low and high frequency limiting values of $\varepsilon^{\prime}$ for the process under investigation. The quantity $C(t)$ is the normalized dipole-dipole correlation function. For non-zero dipole moment perpendicular to the chain contour, the response function $C(t)$ is sensitive to segmental motion.

A Solatron-Schlumberger frequency response analyzer FRA 1260 supplemented by using a high-impedance preamplifier of variable gain was used for the dielectric measurements covering the frequency range $10^{-2}$ to $10^{6} \mathrm{~Hz}$. The sample was pressed in form of a pellet and was residing between two gold-plated stainless steel electrodes (diameter $25 \mathrm{~mm}$ ). It was kept in a cryostat with its temperature controlled via a high-pressure nitrogen gas jet heating system with a Novocontrol Quatro controller allowing a stability of the sample temperature in margins of \pm 0.1 úC in a broad temperature range of -160 úC to +300 úC. Note that the absolute values of the loss part of the dielectric permitivity, $\varepsilon^{\prime \prime}$, depend on the accuracy of the sample thickness provided to the software.

For quantitative analysis, the generalized relaxation function according to HavriliakNegami [26] is traditionally being used; the absence of a clear physical interpretation of the adjustable parameters involved and the fitting uncertainties restrict its use solely as a phenomenological way to account for the features of the underlying processes. Besides, an additional conductivity contribution at low-frequencies and high temperatures due to free charges has to be accounted for. For multiple relaxation processes, use of more than one empirical Havriliak-Negami functions is required; this, however, leads to a large number of adjustable parameters in the fitting procedure (4 parameters are needed for each Havriliak-Negami function). Recently, we have presented a method [27] based on a modification of the widely used CONTIN routine of analysis of photon correlation spectroscopy data for the inversion of the experimental correlation function in order to obtain the distribution of relaxation times $F(\ln \tau)$ with no a priori assumption of the form of the relaxation function. The method performs an inversion of the experimental $\varepsilon^{\prime \prime}(\omega)$ spectrum in order to determine $F(\ln \tau)$ assuming a superposition of Debye processes, i.e.,

$$
\varepsilon^{\prime \prime}(\omega) / \Delta \varepsilon=\int_{-\infty}^{\infty} F(\ln \tau)\left\{\omega \tau /\left[1+(\omega \tau)^{2}\right]\right\}(\ln \tau)
$$

where $F(\ln \tau)$ is normalized. Alternatively, eq. 2a may be written as

$$
\varepsilon^{\prime \prime}(\omega)=\int_{-\infty}^{\infty} \tilde{F}(\ln \tau)\left\{\omega \tau /\left[1+(\omega \tau)^{2}\right]\right\}(\ln \tau)
$$

where $\tilde{F}(\ln \tau)=\Delta \varepsilon F(\ln \tau)$; integration of the resulting $\tilde{F}(\ln \tau)$ spectrum yields $\Delta \varepsilon$, since the $F(\ln \tau)$ distribution is normalized. 


\section{RESULTS AND DISCUSSION}

Figure 1 shows a semilog plot of the loss part of the dielectric permitivity, $\varepsilon^{\prime \prime}(\omega)$, versus frequency for the bulk PMPS sample in the frequency range $10^{-2}$ to $3 \times 10^{6} \mathrm{~Hz}$ for temperatures $223 \mathrm{~K}$ to $253 \mathrm{~K}$. In this range the segmental motion of the PMPS homopolymer $\left(T_{g}=223 \mathrm{~K}\right)$ is observed. This process shows strong temperature dependence in this range (see Figure 4 below), which can be analyzed with a Vogel-Fulcher-Tamman (VFT) equation.

Figure 2 shows the dielectric spectra for the organically-modified silicate B34 in the temperature range of 173 to $223 \mathrm{~K}$. The dielectric spectra of the silicate (both of B34 and B38) are broad and quite complicated. Inversion of the dielectric data using eq. 2 results in two individual relaxation processes for each silicate (see Figure 4 below); the faster one observed in the range of (e.g., Fig. 2) should be related to orientational motions of the alkylammonium surfactant used to modify the silicate surface whereas the slower one observed in the temperature range 275 to $335 \mathrm{~K}$ (not shown) is most probably related to the Maxwell-Wagner polarization due to the presence of interfaces in the system.

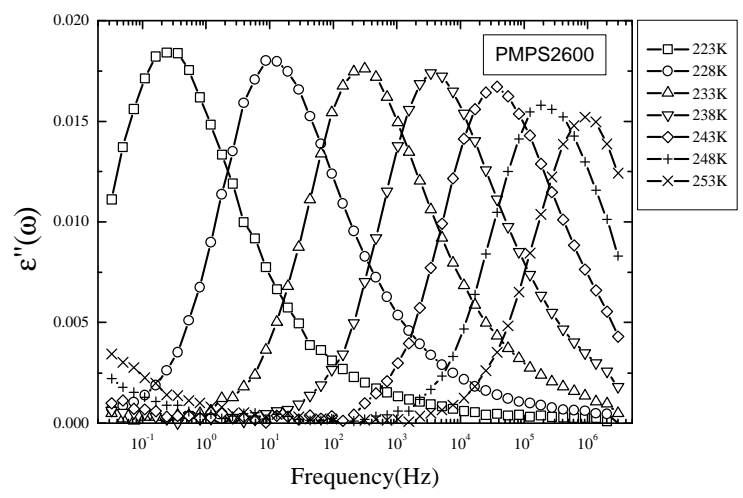

Fig. 1 Frequency dependence of the dielectric loss $\varepsilon$ " for bulk PMPS for temperatures 223-253 K.

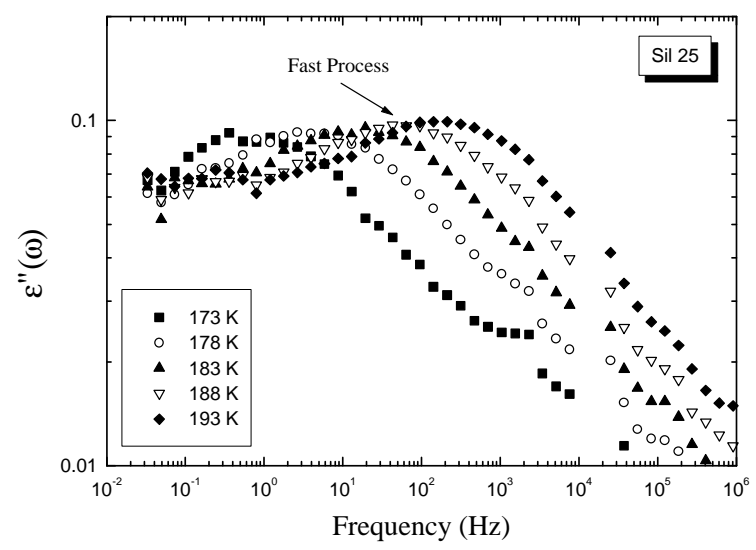

Fig. 3 Frequency dependence of the dielectric loss $\varepsilon$ " for 25\% PMPS/B38 intercalated silicate for temperatures 173-193 K.

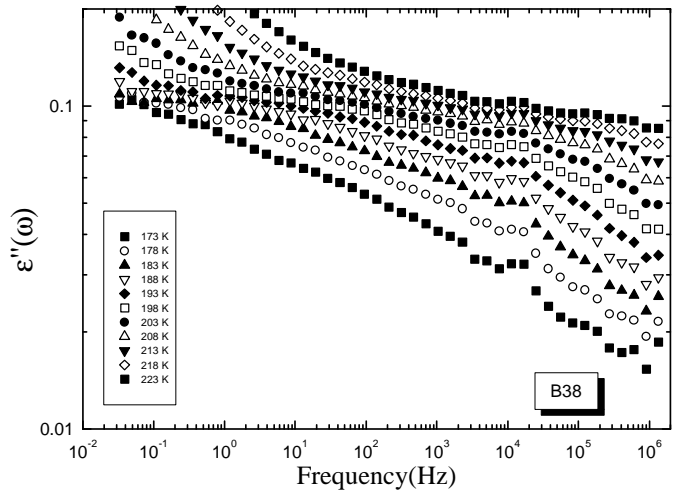

Fig. 2 Frequency dependence of the dielectric loss $\varepsilon^{\prime \prime}$ for the organically modified silicate B38 for temperatures $173-223 \mathrm{~K}$.

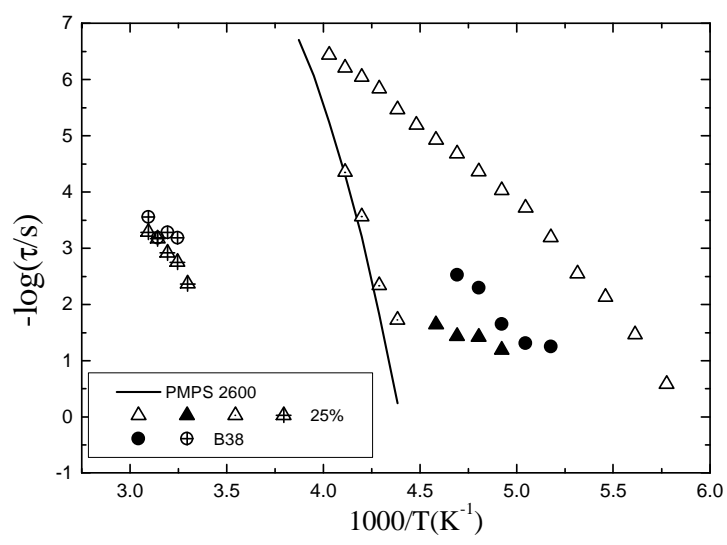

Fig. 4 Arrhenius map for neat PMPS, organically modified silicate B38 and the $25 \%$ intercalated silicate. 
Figure 3 shows the dielectric spectra for the 25\% PMPS/B38 sample in the temperature range $173 \mathrm{~K}$ to $193 \mathrm{~K}$. In this range a very fast process one having weak temperature dependence is observed dependence, which was not observed in either pure PMPS or pure B38 silicate. Besides, three more processes can be identified: one with characteristic times in the same range with the fast process observed in the B38 silicate, which should correspond to the silicate itself; a process with very weak amplitude which superimposes on the relaxation of the pure PMPS, which should correspond to PMPS homopolymer which is not intercalated within the galleries; and one with relaxation times similar to those for the Maxwell-Wagner polarization observed in the B38 silicate. Actually the dielectric strength of the PMPS-like process is extremely small in the $15 \%$ PMPS/B34 system, which is apparently starved.

Figure 4 shows an Arrhenius plot of the most probable relaxation times obtained from the peaks values of the distributions $\tilde{F}(\ln \tau)$ for the $25 \%$ PDMS/B38 specimen as a function of temperature. What is really surprising is the existence of the very fast process, which is observed at temperatures much lower than the glass transition temperature of pure PMPS (also observed in the other systems). It should be noted that such a process is not observed in the neat organicallymodified silicates. Actually, this process has a very weak temperature dependence, which, when fitted with the VFT equation leads to an effective "glass transition temperature" which is almost 60 degrees lower than the PMPS $T_{g}$ (the fast process for the 15\% PMPS/B34 sample has an even weaker temperature dependence). This very fast reorientational motion can only be attributed to the PMPS, which is confined within the galleries of the intercalated silicate. Note that this dynamics appears to merge with that for bulk PMPS at high temperatures. Such fast dynamics can be understood in terms of the cooperativity of segmental reorientation. At high temperatures, the range of cooperativity is smaller than the interlayer spacing; thus, no difference can be expected between the bulk PMPS and that confined within the galleries. As the temperature decreases, the $\alpha$-dynamics deviate from the Arrhenius temperature dependence due to the involvement of cooperativity. The characteristic correlation length increase with decreasing temperature apparently until it becomes equal to the interlayer spacing; this has been termed "hindered glass transition" [28]. In the bulk PMPS, the cooperativity length increases unhindered and, thus, the dynamics is increasingly retarded.

A similar observation of faster dynamics has been made [20] for the low-molecular weight glass-forming liquid salol confined in nanopores $(2.5,5.0$, and $7.5 \mathrm{~nm}$ ), where, however, the observed effect was much smaller. Arguments on cooperativity lead to an estimation for the length scale of cooperativity greater than $7 \mathrm{~nm}$ in the vicinity of the calorimetric glass transition. It should be noted that the effective confinement in that case is much smaller than the one in the present system. Here the interlayer thickness that is available to the polymer chain is about 1.5$2.0 \mathrm{~nm}$, which is of the order of a few statistical segment lengths of the PMPS molecule. Therefore, one may envision laterally segregated chains where the re-orientational motion takes place within a few polymer segments.

A question one may pose is what is the effect of the organic modifier, which is also present in the galleries, on the PMPS segmental motion. One may simply think that a mixing may occur between PMPS and the octadecyl chains of the surfactant modifier used. It has been reported [20] that, because of the lubricant coating on the inner surface of the porous material, the cooperatively rearranging molecules in the pores are not blocked and may reorient within the pore volume. To address this question, we have studied binary mixtures of PMPS with the amine derivative of the surfactant used as organic modifier, i.e, with N,N-dimethyl-1octadecanamine, where the weight fractions polymer:amine were varied from 90:10 to 62:38 (this is estimated to be the ratio of polymer to surfactant within the gallery). It should be noted 
that the phase state of such mixtures is unknown since the amine may not want to mix with PMPS. For the 90:10 mixture, only one process is clearly observed, which superimposes nicely with that of neat PMPS. For the 62:38 specimen, however, two processes are observed: one corresponding to neat PMPS and one that is even faster than the fast process in Figure 4; actually the later process falls on top of the one for the 15\% PMPS/B34 samples at the lowest temperatures investigated. This finding and whether it is related to the faster process observed in all the silicates is under further investigation. Note, that if this is due to a phase separated system of PMPS and amine, i.e., that the fast process is due to amine-rich regions in the system, this should not influence the behavior of the intercalated silicate, since the PMPS and the surfactant are in close (molecular) proximity due to the confinement.

\section{CONCLUDING REMARKS}

Dielectric Relaxation Spectroscopy has been utilized to probe local dynamics in a series of nanocomposites consisting of poly(methyl-phenyl-siloxane) (PMPS) intercalated in organically modified silicates, with varying polymer content. As verified from X-Ray Diffraction measurements, the material is confined in spaces of 15-20 ${ }^{1}$. The effect of confinement is directly reflected on the conformational behavior associated to local reorientational dynamics, as implied by the observation of a new mode faster from the segmental $\alpha$-relaxation of the bulk material and characterized by a weaker temperature dependence. Interpretation of the observed behavior in terms of the current view in local dynamics is discussed. 


\section{ACKNOWLEDGMENTS}

S. H. A. acknowledges that part of this research was sponsored by NATO's Scientific Affairs Division in the framework of the Science for Stability Programme and by the Greek General Secretariat of Research and Technology.

\section{REFERENCES}

1. E. P. Giannelis, Advanced Materials 8, 29 (1996).

2. R. K. Krisnamoorti, R. A. Vaia, and E. P. Giannelis, Chem. Mat. 8, 1728 (1996)

3. T. Lan, T. Pinnavaia, Chem. Mater. 2, 2216 (1994); Chem. Mater. 7, 2144 (1995).

4. Y. Kojima, A. Usuki, M. Kawasumi, A. Okada, T. Kurauchi, O. Kaamigaito, J. Polym. Sci. A 31, 983 (1993). Y. Kojima, J. Mater. Res. 8, 1185 (1993).

5. S. D. Burnside, and E. P. Giannelis, Chem. Mater. 7, 1597 (1995).

6. J. D. Lee, T. Takekoshi, and E. P. Giannelis, Mater. Res. Soc. Symp. Proc. 457, 513 (1997).

7. E. P. Giannelis, R. Krisnamoorti, and E. Manias, Adv. Pol. Sci., in press.

8. $\quad$ J. L. Keddie, R. A. L. Jones, and R. A. Covy, Europhys. Lett. 27, 59 (1994).

9. G. Reiter, Europhys. Lett. 23, 579 (1993).

10. K. F. Mansfield, and D. N. Theodorou, Macromolecules 24, 6283 (1991).

11. J. L. Keddie, R. A. L. Jones, and R. A. Covy, Faraday Disc. 98, 219 (1994).

12. J. H. van Zanten, W. E. Wallace, and W. Wu, Phys. Rev. E 53, R2053 (1996).

13. X. Zheng, B. B. Sauer, J. G. van Alsten, S. A. Schwarz, M. H. Rafailovich, J. Sokolov, and M. Rubinstein, Phys. Rev. Lett. 74, 407 (1995). X. Zheng, M. H. Rafailovich, J. Sokolov, Y. Strzhemechny, S. A. Schwarz, B. B. Sauer, and M. Rubinstein, Phys. Rev. Lett. 79, 241 (1997).

14. J. Baschnagel, and K. Binder, J. Phys. I France 6, 1271 (1996)

15. J. A. Forrest, K. Dalnoki-Veress, J. R. Stevens, and J. R. Ducher, Phys. Rev. Lett. 77, 2002 (1996).

16. C. L. Jackson, and G. B. McKenna, J. Chem. Phys. 93, 9002 (1990).

17. G. Liu, Y. Li, and J. Jonas, J. Chem. Phys. 95, 6892 (1991). S. Stapf, R. Kimmich, and R.-O. Seitter, Phys. Rev. Lett. 75, 2855 (1995).

18. R. Richert, Phys. Rev. B 54, 15762 (1996).

19. Schüller, Yu. B. Mel'nichenko, R. Richert, and E. W. Fischer, Phys. Rev. Lett. 73, 2224 (1994).

20. M. Arndt, R. Stannarius, H. Groothues, E. Hempel, and F. Kremer, Phys. Rev. Lett. 79, 2077 (1997)

21. A. Huwe, M. Arndt, F. Kremer, G. Haggenmüller, and P. Behrens, J. Chem. Phys. 107, 9699 (1997)

22. L. Petychakis, G. Floudas, and G. Fleischer, Europhys. Lett. 40, 685 (1997).

23. B. Jérôme, and J. Commandeur, Nature 386, 589 (1997).

24. G. Barut, P. Pissis, R. Pelster, and G. Nimitz, Phys. Rev. Lett. 80, 3543 (1998).

25. R. A. Vaia, R. K. Teukolsky, and E. P. Giannelis, Chem. Mater. 6, 1017 (1994). R. A. Vaia, and E. P. Giannelis, Macromolecules 30, 7990 (1997); 30, 8000 (1997).

26. S. Havriliak, and S. Negami, Polymer 8, 161 (1967).

27. K. Karatasos, S. H. Anastasiadis, A. N. Semenov, G. Fytas, M. Pitsikalis, and N. Hadjichristidis, Macromolecules 27, 3543 (1994).

28. E. Donth, Glasübergang (Akademie Verlag, Berlin, 1981). 